\title{
Forming Simulation Considering the Differential Work Hardening Behavior of a cold rolled interstitial-free steel Sheet
}

\author{
Kazuhiro Ichikawa ${ }^{1, a}$, Toshihiko Kuwabara ${ }^{1, b}$, Sam Coppieters ${ }^{2, c}$ \\ ${ }^{1}$ Graduate school, Department of Mechanical Systems Engineering, Tokyo University of Agriculture \\ and Technology, 2-24-16, Nakacho, Koganei-shi, Tokyo, 184-8588, Japan \\ ${ }^{2}$ Department of Metallurgy and Materials Engineering, KU Leuven, Campus Gent, \\ Gebroeders De Smetstraat 1, 9000 Gent, Belgium
}

a50010255011@st.tuat.ac.jp, buwabara@cc.tuat.ac.jp, Sam.Coppieters@mtm.kuleuven.be

Keywords: yield locus, yield function, cruciform specimen, multiaxial tube expansion test, biaxial stress, digital image correlation

\begin{abstract}
The multiaxial plastic deformation behavior of a cold rolled interstitial-free steel sheet with a thickness of $0.65 \mathrm{~mm}$ was measured using a servo-controlled multiaxial tube expansion testing machine for the range of strain from initial yield to fracture. Tubular specimens were fabricated from the sheet sample by roller bending and laser welding. Many linear stress paths in the first quadrant of stress space were applied to the tubular specimens to measure the contours of plastic work in stress space up to an equivalent plastic strain of 0.289 along with the directions of plastic strain rates. The test material exhibited differential hardening (DWH). A material modeling method for reproducing the DWH in a finite element simulation has been developed. Hydraulic bulge forming simulation results based on the DWH model had a closer agreement with the experimental results than those calculated using the isotropic hardening models with selected yield functions.
\end{abstract}

\section{Introduction}

In sheet metal forming simulations the effect of yield functions on the predictive accuracy of material deformation behavior is significant $[1,2]$. One of the authors developed a biaxial tensile testing method for sheet metals using a cruciform specimen [3,4] and demonstrated that it is necessary to select appropriate yield functions for the sheet samples to obtain accurate finite element analysis (FEA) results for hole expansion [5, 6], shallow shell drawing of an automotive body panel [7] and hydraulic bulge forming [8].

One of the authors investigated the work hardening behavior of pure titanium sheet using biaxial tensile tests using cruciform specimens and tubular specimens [9]. Tubular specimens were fabricated by bending the sheet sample into a constant curvature and welding the sheet edges together, and combined internal pressure and axial load were applied to the tubular specimens using a servo-controlled multiaxial tube expansion testing machine [10]; henceforth, we refer to this testing method as Multiaxial Tube Expansion Testing method (MTET). Consequently, contours of plastic work in a principal stress space were successfully measured up to a work equivalent plastic strain, $\varepsilon_{0}^{\mathrm{p}}$, of 0.085 , while $\varepsilon_{0}^{\mathrm{p}}$ was only 0.002 for the biaxial tensile tests using cruciform specimens. More recently, the MTET was applied to a precipitation hardening steel sheet with a tensile strength of 590 $\mathrm{MPa}$, and contours of plastic work were successfully measured for a strain range of $\varepsilon_{0}^{\mathrm{p}} \leq 0.16$ [11].

In these MTET experiments the tests had to be interrupted several times to change strain gauges. In order to overcome this problem, the author's research group has developed a new strain measurement apparatus using extensometers for tubular specimens [12]. Many linear stress paths in the first quadrant of stress space were applied to the tubular specimens made of a ultralow carbon steel sheet, and the forming limit curve (FLC) and forming limit stress curve (FLSC) were successfully measured.

In this study, the MTET is applied to a cold rolled interstitial-free steel sheet. A material model for reproducing the differential work hardening (DWH) of the test sample in a finite element analysis (FEA) has been developed. In addition, hydraulic bulge forming experiments and simulations has been performed to investigate the effects of material models on the accuracy of the forming simulation results. 


\section{Experimental Methods}

Test Material. The test material used in this study was a cold rolled interstitial-free steel sheet (SPCD in JIS) with a thickness of $0.65 \mathrm{~mm}$. The work hardening characteristics and $r$-values at $0^{\circ}$, $45^{\circ}$ and $90^{\circ}$ (transverse direction; TD) to the rolling direction (RD) are listed in Table 1 . The RD, TD and the thickness directions of the material are defined as the $x-, y$ - and $z$-axes, respectively.

Table 1 Mechanical properties of the test material (SPCD)

\begin{tabular}{lccccc}
\hline Tensile direction $\left[{ }^{\circ}\right]$ & $\sigma_{0.2}[\mathrm{MPa}]$ & $c^{*}[\mathrm{MPa}]$ & $n^{*}$ & $\alpha^{*}$ & $r$-value** \\
\hline $0(x)$ & 158 & 541 & 0.249 & 0.0036 & 1.34 \\
45 & 162 & 550 & 0.262 & 0.0053 & 1.13 \\
$90(y)$ & 159 & 535 & 0.260 & 0.0051 & 1.50 \\
\hline *Parameters for Swift's hardening law, $\sigma=c\left(\alpha+\varepsilon^{\mathrm{p}}\right)^{n}$, for a strain range of $0.002 \leq \varepsilon^{\mathrm{p}} \leq 0.248\left(0^{\circ}\right), 0.254\left(45^{\circ}\right)$, \\
0.259 $\left(90^{\circ}\right)$. \\
** Measured at nominal strain $\varepsilon_{\mathrm{N}}=0.10$.
\end{tabular}

Specimens for Biaxial Tensile Tests. Two types of biaxial tensile tests were performed to measure the plastic deformation behavior of the test material from initial yield up to fracture. Fig. 1 (a) shows a schematic of the cruciform specimen used for the biaxial tensile test of the as-received sheet sample. The geometry of the specimen was the same as that used in [3]. The specimen arms were parallel to the RD and TD of the material. The slits were fabricated by laser cutting.

Normal strain components $\left(\varepsilon_{x}, \varepsilon_{y}\right)$ were measured using uniaxial strain gauges (YFLA-2, Tokyo Sokki Kenkyujo Co.) mounted at $\pm 21 \mathrm{~mm}$ from the center along the maximum loading direction. According to the FEA of the cruciform specimen with the strain measurement position shown in Fig. 1(a), the stress measurement error was estimated to be less than 2\% [13][14]. Details of the biaxial testing apparatus and test method are given in [3].

Fig. 1(b) schematically shows the tubular specimen used for the multiaxial tube expansion tests. The specimens were fabricated by bending the sheet sample into a cylindrical shape with an inner diameter of $54 \mathrm{~mm}$ and laser-welding the sheet edges together. Two types of tubular specimens were fabricated; type I specimen had the RD in the axial direction and type II specimen had the RD in the circumferential direction. Type I specimens were used for tests with $\sigma_{x}<\sigma_{y}$ and type II for tests with $\sigma_{x} \geq \sigma_{y}$; the maximum principal stress direction was always taken to be in the circumferential direction.

Experimental Apparatus for Multiaxial Tube Expansion Test. Fig. 2 (left) shows a schematic diagram of the servo-controlled tension-internal pressure testing machine developed by the author's research group. An axial load $T$ and internal pressure $P$ are applied to a tubular specimen by a hydraulic cylinder and a pressure booster, respectively, and the circumferential and axial strains, $\varepsilon_{\theta}$

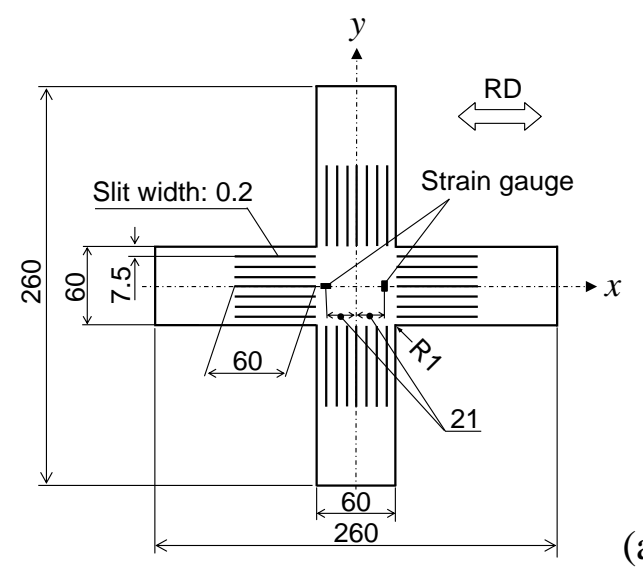

(a)

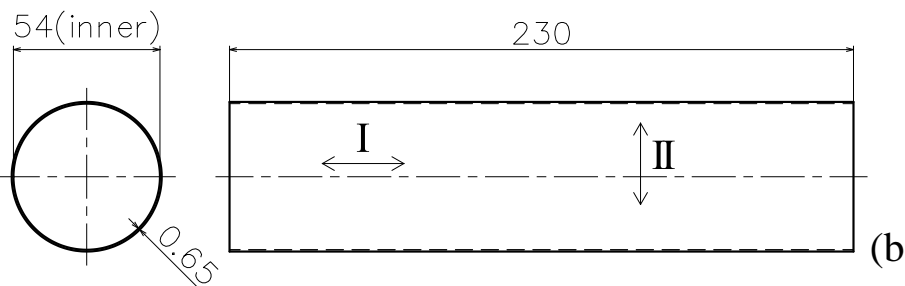

Fig. 1 Specimens used for the biaxial tensile tests (dimensions in $\mathrm{mm}$ ): (a) cruciform specimen and (b) tubular specimen. $\leftrightarrow$ indicates the RD of the original sheet sample. 


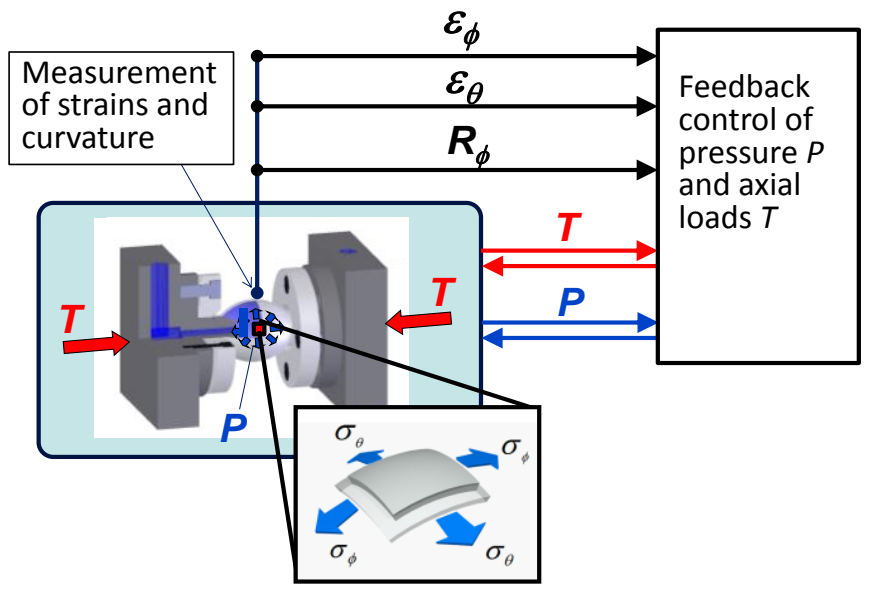

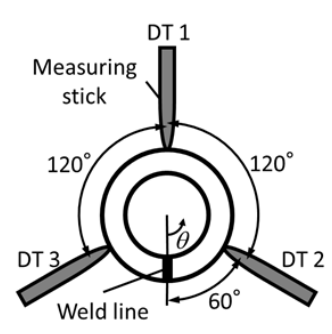

(a)

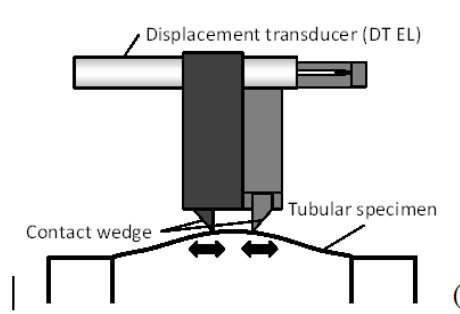

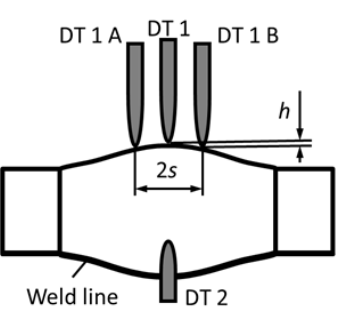

(b)

Fig. 2 (Left) A schematic diagram of the servo-controlled tension-internal pressure testing machine used for multiaxial tube expansion test. (Right) Schematic diagram of the strain measurement apparatus used for the multiaxial tube expansion test method [12]. Sensors for measuring (a) circumferential strain $\varepsilon_{\theta}$, (b) the radius of curvature $R_{\phi}$ in the axial direction, and (c) axial strain $\varepsilon_{\phi}$.

and $\varepsilon_{\phi}$, at the mid-section of the bulging specimen and the radius of axial curvature $R_{\phi}$ are measured simultaneously. The axial and circumferential stresses, $\sigma_{\phi}$ and $\sigma_{\theta}$, at the mid-section of the bulging specimen can be calculated as the values at the mid wall using the following equations based on the equilibrium requirements at the center of the specimen:

$$
\begin{aligned}
\sigma_{\phi} & =\frac{P \pi(D / 2-t)^{2}+T}{\pi(D-t) t}, \\
\sigma_{\theta} & =\frac{\left(R_{\phi}-t\right)(D-2 t)}{\left(2 R_{\phi}-t\right) t} P-\frac{D-t}{2 R_{\phi}-t} \sigma_{\phi},
\end{aligned}
$$

Fig. 2 (right) shows schematic diagrams of the experimental apparatus for measuring the circumferential strain $\varepsilon_{\theta}$, the radius of axial curvature $R_{\phi}$ at the bulging area, and the axial strain $\varepsilon_{\phi}$ at the mid-section of the bulging specimen to enable continuous measurement of the biaxial stress-strain curves, $\sigma_{\phi}-\varepsilon_{\phi}$ and $\sigma_{\theta}-\varepsilon_{\theta}$, of a tubular specimen for a large strain range. The details of the feedback circuit for controlling the true stress paths can be found in [12].

Measurement of Contours of Plastic Work. Both cruciform and tubular specimens were subjected to proportional loading with true stress ratios $\sigma_{x}: \sigma_{y}=4: 1,2: 1,4: 3,1: 1,3: 4,1: 2$ and 1:4. A standard uniaxial tensile specimen (JIS 13 B-type) was used for the uniaxial tensile tests with $\sigma_{x}: \sigma_{y}=1: 0$ and $0: 1$. True stress increments were controlled and applied to the specimens so that the von Mises equivalent plastic strain rate became approximately constant at $5 \times 10^{-4} \mathrm{~s}^{-1}$ for all stress paths. Two specimens were used for each stress ratio.

The concept of the contour of plastic work in stress space $[15,16]$ was introduced in order to evaluate the work hardening behavior of the test material under biaxial tension. The stress-strain curve obtained from a uniaxial tensile test along RD was selected as a reference datum for work hardening; the uniaxial true stresses $\sigma_{0}$ and the plastic work per unit volume $W_{0}$ corresponding to particular values of offset true plastic strains $\varepsilon_{0}^{\text {p }}$ were determined. The uniaxial true stress $\sigma_{90}$ obtained from a tensile test in TD and the biaxial true stress components $\left(\sigma_{x}, \sigma_{y}\right)$ obtained from biaxial tensile tests were then determined at the same plastic work as $W_{0}$. The stress points $\left(\sigma_{0}, 0\right)$, $\left(0, \sigma_{90}\right)$ and $\left(\sigma_{x}, \sigma_{y}\right)$ thus plotted in the principal stress space comprise a contour of plastic work corresponding to a particular value of $\varepsilon_{0}^{\mathrm{p}}$.

The stress-strain curves measured from the biaxial tensile tests of the tubular specimens were offset and smoothly connected to those measured using the cruciform specimens to compensate the effect of prestrain caused by bending, using the same method as described in [12]. 


\section{Experimental Results of Biaxial Stress Tests}

Fig. 3 shows the measured stress points forming contours of plastic work for different levels of $\varepsilon_{0}^{\mathrm{p}}$; in Fig. 3(b) the stress values are normalized by $\sigma_{0}$ associated with the specific value of $\varepsilon_{0}^{\mathrm{p}}$. For $\sigma_{x}: \sigma_{y}=1: 1$, fracture occurred at a weld line of the tubular specimens; therefore, a hydraulic bulge test was performed to measure the work hardening behavior for the strain range of $\varepsilon_{0}^{\mathrm{p}} \geq 0.13$. Also depicted in the figures are the theoretical yield loci based on the von Mises, Hill's quadratic (Hill '48) [16], and the Yld2000-2d yield function with exponents of $M$ [17]. The work contours normalized by $\sigma_{0}$ show a tendency of expansion in the direction of $\sigma_{x}: \sigma_{y}=1: 1$ with the increase of $\varepsilon_{0}^{\mathrm{p}}$ for $\varepsilon_{0}^{\mathrm{p}} \leq 0.20$, while the shapes are almost similar (isotropic hardening) for $\varepsilon_{0}^{\mathrm{p}}>0.15$.

Fig. 4 shows the variation of the material parameters $\alpha_{i}$ and exponent $M$ of the Yld2000-2d yield function with $\varepsilon_{0}^{\mathrm{p}}$. The values of $\alpha_{i}$ and $M$ show a relatively large variation for $\varepsilon_{0}^{\mathrm{p}} \leq 0.03$, while they are almost constant (isotropic hardening) for $\varepsilon_{0}^{\mathrm{p}}>0.03$.

\section{Hydraulic Bulge Forming Experiment and Finite Element Simulation}

Hydraulic bulge tests were performed to quantitatively evaluate the effect of the material models on the predictive accuracy of sheet metal forming simulations. Fig. 5(a) shows the experimental

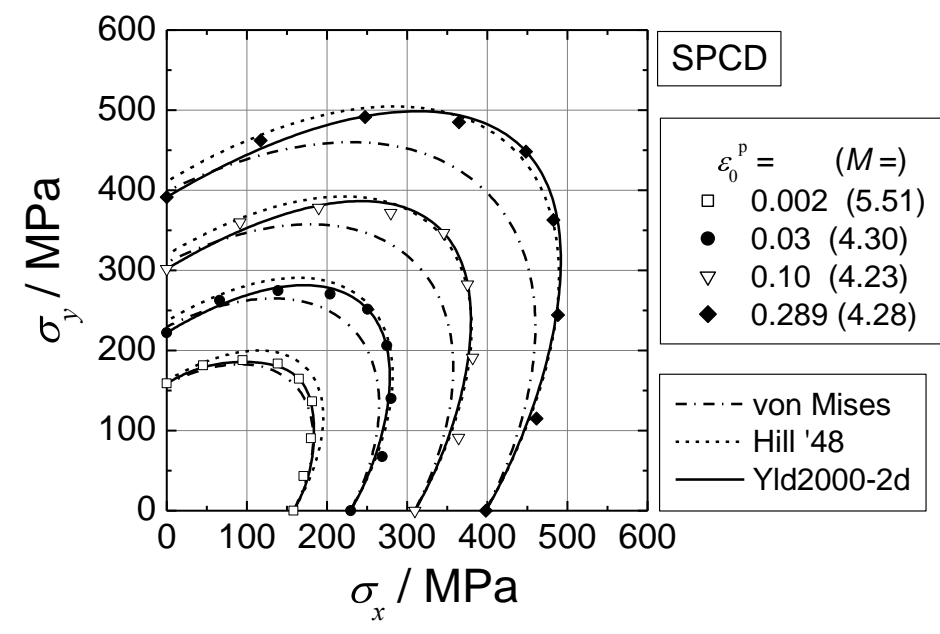

(a)

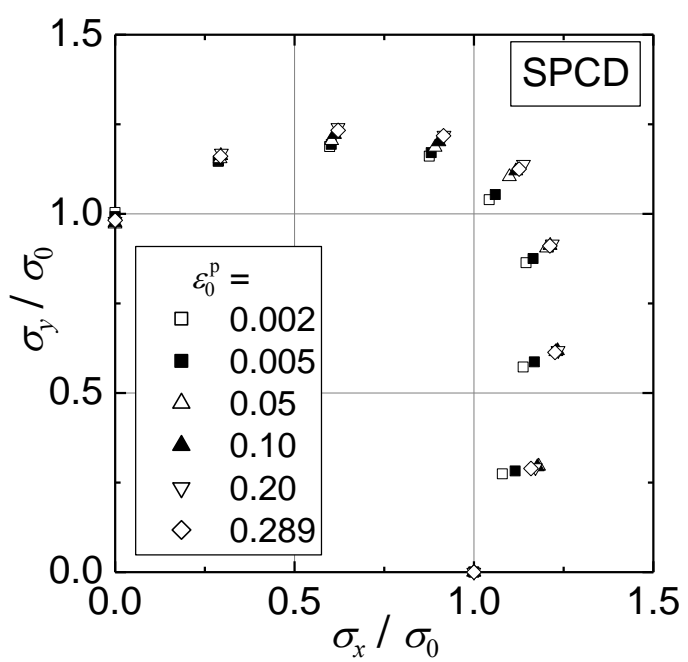

(b)

Fig. 3 (a) Measured stress points forming contours of plastic work for different levels of $\varepsilon_{0}^{\mathrm{p}}$, and (b) those normalized by the uniaxial tensile flow stress $\sigma_{0}$ associated with $\varepsilon_{0}^{\mathrm{p}}$.

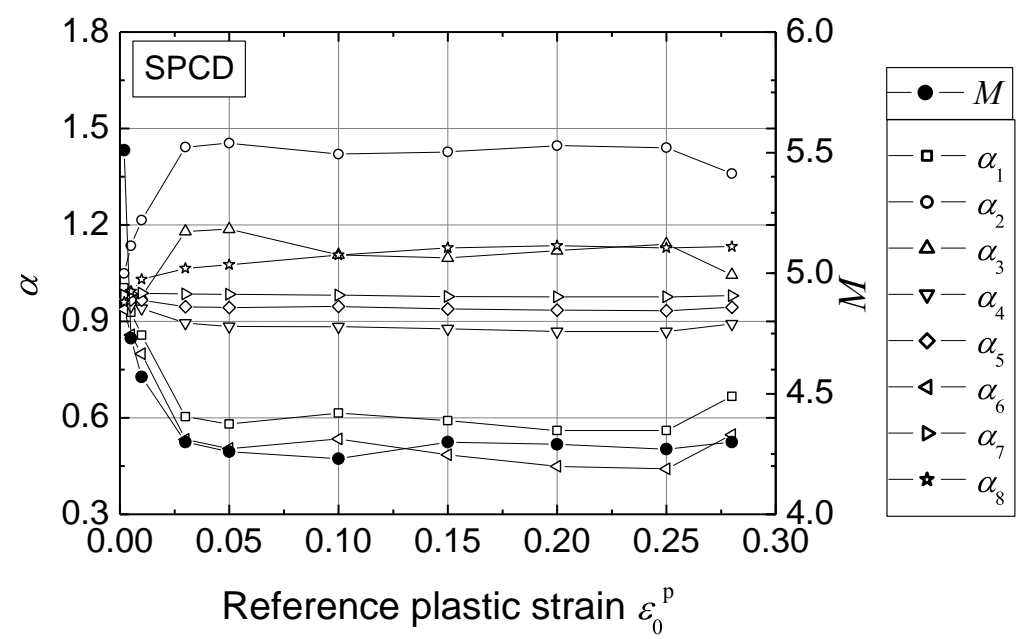

Fig. 4 Variation of the material parameters $\alpha_{i}$ and an exponent $M$ of the Yld2000-2d yield function with $\varepsilon_{0}^{\mathrm{p}}$. 
apparatus used for the hydraulic bulge forming. The diameter of the die opening was $150 \mathrm{~mm}$, the die profile radius was $8 \mathrm{~mm}$, and the blank diameter was $220 \mathrm{~mm}$. The material flow-in was fixed at zero along the boundary of a $190 \mathrm{~mm}$ diameter using a triangular draw-bead. No lubricant was used at the interface between the blank and die surface.

Using IVIEW which is an application software attached to ARAMIS ${ }^{\circledR}$, measured data can be incorporated into the feedback control circuit. In ARAMIS ${ }^{\circledR}$, nominal strains for each direction are calculated using the distance between the two facets, whose initial distance (gauge length) is about 15 $\mathrm{mm}$. The hydraulic pressure $P$ was controlled so that the equivalent plastic strain rate was kept approximately constant, $10^{-4} \mathrm{~s}^{-1}$ ).

FEA simulations of the hydraulic bulge forming were carried out using Abaqus/Standard Ver.6.12-3. One quarter of a circular blank was analyzed due to the orthotropic symmetry of the material. The blank diameter was $190 \mathrm{~mm}$ and the nodal displacement along the periphery of the blank was fixed to zero, because the radial position of the draw-bead in the die used in the experiment was $95 \mathrm{~mm}$. 4-node shell elements, S4R, were used. A surface-to-surface contact condition of the blank to the die was selected with a blank holding force of $60 \mathrm{kN}$ and a friction coefficient of 0.3 .

The yield functions used in the FEA were the von Mises and Hill's quadratic [16] yield functions shown in Fig. 3 (a), and the Yld2000-2d yield function [17] approximating the work contour for $\varepsilon_{0}^{\mathrm{p}}=0.289$ with isotropic hardening assumption and the Yld2000-2d yield function taking account for the DWH of the test sample, see Fig. 4. Work hardening was described by Swift's hardening law (see Table 1) fitted to the available pre-necking data obtained from a standard tensile test in the rolling direction.

\section{Results and discussion}

Fig. 5 (b) shows the measured true thickness strain-pressure curve with the FEA results. The von Mises and Hill' 48 yield functions underestimate the maximum pressure, while the Yld2000-2d yield function with DWH shows the closest agreement with the measurement. The difference of the calculated curves between the Iso and DWH models for the Yld2000-2d yield function is small. This result is consistent with the fact that the test material exhibits isotropic hardening for $\varepsilon_{0}^{\mathrm{p}}>0.03$, as shown in Fig. 4.

The Yld2000-2d yield function slightly underestimates the pressure for a strain range of $0.6 \leq\left|\varepsilon_{z}\right|$. This is possibly due to the fact that the work hardening equation used in the FEA was determined for a strain range of $0.002 \leq \varepsilon^{\mathrm{p}} \leq 0.248$ (see Table 1), which is quite smaller than the total plastic strain,

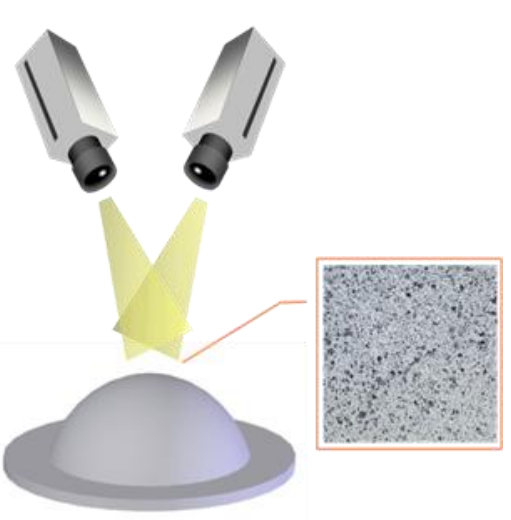

(a)

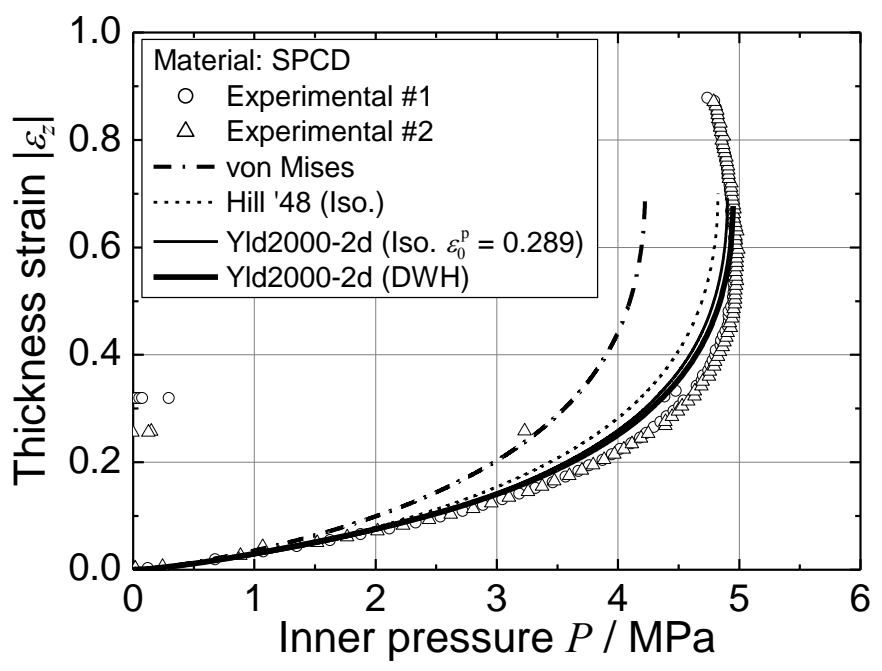

(b)

Fig. 5 (a) A schematic view of the hydraulic bulge test using a digital image correlation system for strain measurement. (b) The measured true thickness strain-pressure curve with the FEA results. 
$0.6 \approx\left|\varepsilon_{z}\right|$, applied to the sheet sample in the bulge forming experiment. An accurate modeling of the DWH of the test material for a strain range of $0.25 \leq \varepsilon^{\mathrm{p}} \leq 0.6$ would be necessary to obtain more accurate FEA results. Work along these lines is currently conducted [18].

\section{References}

[1] T. Kuwabara, Advances in experiments on metal sheets and tubes in support of constitutive modeling and forming simulations, Int. J. Plasticity 23 (2007) 385-419.

[2] D. Banabic, F. Barlat, O. Cazacu, T. Kuwabara, Advances in anisotropy and formability, Int. J. Mater. Form. 3 (2010) 165-189.

[3] T. Kuwabara, S. Ikeda, T. Kuroda, Measurement and analysis of differential work hardening in cold-rolled steel sheet under biaxial tension, J. Mater. Process Technol. 80-81 (1998) 517-523.

[4] T. Kuwabara, A. Van Bael, E. Iizuka, Measurement and analysis of yield locus and work hardening characteristics of steel sheets with different r-values, Acta Mater. 50/14 (2002) 3717-3729.

[5] K. Hashimoto, T. Kuwabara, E. Iizuka, J.-W. Yoon, Effect of anisotropic yield functions on the accuracy of hole expansion simulations for 590MPa grade steel sheet, Tetsu-to-Hagané 96 (2010) 557-563. (in Japanese)

[6] T. Kuwabara, K. Hashimoto, E. Iizuka, J.-W. Yoon, Effect of anisotropic yield functions on the accuracy of hole expansion simulations, J. Mater. Processing Technol. 211 (2011) 475-481.

[7] T. Moriya, T. Kuwabara, S. Kimura, S. Takahashi, Effect of anisotropic yield function on the predictive accuracy of surface deflection of automotive outer panels, Steel Research Int. 81 (2010) 1384-1387.

[8] D. Yanaga, T. Kuwabara, N. Uema, M. Asano, Material modeling of 6000 series aluminum alloy sheets with different density cube textures and effect on the accuracy of finite element simulation, Int. J. Solids and Structures 49 (2012) 3488-3495.

[9] M. Ishiki, T. Kuwabara, Y. Hayashida, Measurement and analysis of differential work hardening behavior of pure titanium sheet using spline function, Int. J. Mater. Forming 4 (2011) 193-204.

[10] T. Kuwabara, K. Yoshida, K. Narihara, S. Takahashi, Anisotropic plastic deformation of extruded aluminum alloy tube under axial forces and internal pressure, Int. J. Plasticity 21 (2005) 101-117.

[11] T. Hakoyama and T. Kuwabara, Biaxial tensile test of high strength steel sheet for large plastic strain range, Key Engineering Materials 504-506 (2012) 59-64.

[12] T. Kuwabara, F. Sugawara, Multiaxial tube expansion test method for measurement of sheet metal deformation behavior under biaxial tension for a large strain range, Int. J. Plasticity 45 (2013), 103-118.

[13] Y. Hanabusa, H. Takizawa, T. Kuwabara, Evaluation of accuracy of stress measurements determined in biaxial stress tests with cruciform specimen using numerical method, Steel Research Int. 81 (2010) 1376-1379.

[14] Y. Hanabusa, H. Takizawa, T. Kuwabara, Numerical verification of a biaxial tensile test method using a cruciform specimen, J. Mater. Processing Technol. 213 (2013) 961-970.

[15] R. Hill, S.S. Hecker, M.G. Stout, An investigation of plastic flow and differential work hardening in orthotropic brass tubes under fluid pressure and axial load, Int. J. Solids Struct. 31 (1994) 2999-3021.

[16] R. Hill, A theory of the yielding and plastic flow of anisotropic metals, Proc. Roy. Soc. London A193 (1948) 281-297.

[17] F. Barlat, J.C. Brem, J.W. Yoon, K Chung, R.E. Dick, D.J. Lege, F. Pourboghrat, S.H. Choi, E. $\mathrm{Chu}$, Plane stress yield function for aluminum alloy sheets - Part 1: Theory, Int. J. Plasticity 19 (2003) 1297-1319.

[18] S. Coppieters, K. Ichikawa, , D. Debruyne, , T. Kuwabara, , Identification of post-necking hardening behaviour of sheet metal: influence of the yield function. ICEM16, Cambridge, UK. 